\title{
Desempenho dos programas de prevenção e controle de infecções em pequenos hospitais*
}

\section{Performance of infection prevention and control programs in small hospitals \\ Desempeño de programas de prevención y control de infecciones en pequeños hospitales}

Como citar este artigo:

Santos PLC, Padoveze MC, Lacerda RA. Performance of infection prevention and control programs in small hospitals. Rev Esc Enferm USP. 2020;54:e03617. doi: https://doi.org/10.1590/S1980-220X2019002103617

Pryscilla Ladislau Carneiro Santos ${ }^{1}$

Maria Clara Padoveze ${ }^{2}$

Rúbia Aparecida Lacerda ${ }^{3}$

* Extraído da dissertação: "Programas de Prevenção e Controle de Infecções relacionadas à assistência à saúde em pequenos hospitais: diagnóstico de situação", Escola de Enfermagem, Universidade de São Paulo, 2016.

${ }^{1}$ Universidade de São Paulo, Escola de Enfermagem, Programa de Pós-

Graduação em Enfermagem em Saúde do Adulto, São Paulo, SP, Brasil.

${ }^{2}$ Universidade de São Paulo, Escola de Enfermagem, Departamento de Enfermagem em Saúde Coletiva, São Paulo, SP, Brasil.

${ }^{3}$ Universidade de São Paulo, Escola de Enfermagem, Departamento de Enfermagem Médico-Cirúrgica, São Paulo, SP, Brasil.

\section{ABSTRACT}

Objective: To evaluate the structure compliance and prevention and control processes of Healthcare-Associated Infections (HAIs). Method: A prospective and crosssectional study conducted from 2015 to 2016 in small hospitals with up to 70 beds in a region of São Paulo state. Four previously validated indicators were evaluated and expressed as a compliance index (percentage in relation to the evaluated items). Results: Fourteen (14) among the 27 recruited hospitals consented to participate in the study. The average compliance values for each indicator were: Program structure (61.0\%); Operational guidelines (84.5\%); Epidemiological surveillance (57.9\%); and Prevention activities (74.5\%). Greater compliance was observed in private hospitals (73.9\%) and with the presence of an intensive care unit (90.3\%). The hospitals had nurses assigned to the program (92.9\%), but only $23.1 \%$ of the private institutions worked exclusively for six hours. Conclusion: Only the indicator referring to the Operational Guidelines of the evaluated programs was above $90 \%$ compliance for the median of hospitals. The greatest dispersion of compliance results among the studied hospitals was related to the Epidemiological Surveillance indicator.

\section{DESCRIPTORS}

Cross Infection; Infection Control; Health Status Indicators; Health Evaluation. 


\section{INTRODUÇÃO}

A assistência à saúde é desafiada por eventos adversos evitáveis, dentre eles, as Infecções Relacionadas à Assistência à Saúde (IRAS), que constituem grave problema de saúde pública mundial, aumentando a mortalidade e elevando os custos hospitalares ${ }^{(1)}$. As condições para prevenção e controle de IRAS têm sido apresentadas como parâmetro de avaliação dos serviços de saúde e reflexo da qualidade dos cuidados prestados $^{(2)}$. A insuficiência destas condições tem sido um desafio especial para os países de baixa e média renda, para os quais os índices de IRAS são ainda maiores ${ }^{(3)}$.

Pequenos hospitais, com 70 leitos ou menos, além de serem predominantes em muitos países como o Brasil, possuem papel particularmente importante em localidades com limitadas estruturas de assistência em cuidados agudos. Isso demonstra a relevância destas instituições, que representam $62 \%$ das unidades hospitalares e $18 \%$ dos leitos existentes no sistema de saúde brasileiro, sendo que mais da metade destes se configuram como a única opção de assistência em muitas localidades ${ }^{(4)}$.

Escassos estudos apresentam dados de IRAS em pequenos hospitais ${ }^{(5-7)} \mathrm{e}$ a magnitude do problema é pouco conhecida no país. Estudo com uma amostra nacional com estratificação segundo o porte hospitalar evidenciou uma taxa total de IRAS de 10,8\%. Para o estrato dos hospitais de pequeno porte foram considerados aqueles entre 10 a 50 leitos; neste grupo foram incluídos 412 pacientes, dentre os quais 5,5\% apresentaram IRAS. Nesse estudo houve predominância da infecção do trato urinário, representando $1,5 \%$ dos casos. Dentre os pacientes que realizaram algum procedimento cirúrgico, identificou-se uma taxa de 4,3\% de infecção do sítio cirúrgico ${ }^{(8)}$. Contudo, os autores destacaram que a maioria das infecções do sítio cirúrgico foram detectadas após a alta e assim sendo, estudos de prevalência tendem a subestimar as taxas deste tipo de infecção.

Com relação às práticas de prevenção e controle de IRAS nestas instituições de pequeno porte, essa ainda é uma realidade pouco conhecida no mundo. Raros são os estudos que abordam as condições de estrutura para a prevenção e controle de IRAS ${ }^{(9-10)}$.

Conhecer a realidade destas instituições de pequeno porte é o primeiro passo para identificar aspectos relevantes para direcionar as políticas públicas voltadas para a qualidade da assistência à saúde em países em desenvolvimento. A fim de oferecer contribuição para o reconhecimento da magnitude deste problema, este estudo objetivou avaliar o índice de conformidade de estrutura e processos dos Programas de Prevenção e Controle de Infecções Relacionadas à Assistência à Saúde (PPCIRAS) de pequenos hospitais em uma região do estado de São Paulo, Brasil.

\section{MÉTODO}

\section{TIPO DE ESTUDO}

Estudo transversal e prospectivo, de avaliação de desempenho de pequenos hospitais no que se refere a estrutura e processo dos PPCIRAS.

\section{Cenário}

Desenvolvido em pequenos hospitais pertencentes à região de Taubaté, localizado no sudeste do estado de São Paulo, Brasil, no período entre setembro de 2015 a março de 2016. A escolha da região de estudo foi definida por conveniência, porém apresenta características semelhantes a outras regiões do estado. Foi estabelecido como critério de inclusão hospitais com até 70 leitos, gerais e especializados, públicos e privados, extraídos após consulta no Cadastro Nacional dos Estabelecimentos de Saúde (CNES: http://cnes.datasus.gov.br), no ano de 2015. Foram excluídas instituições que não realizavam procedimentos invasivos terapêuticos, resultando em uma população total elegível de 27 hospitais.

\section{Coleta de Dados}

Os dados foram coletados por uma das pesquisadoras, com experiência em PPCIRAS, por meio de inspeção de documentos e entrevistas, utilizando dois instrumentos estruturados. $\mathrm{O}$ primeiro instrumento foi utilizado para caracterização dos hospitais e seus PPCIRAS. O segundo, para avaliação da conformidade dos PPCIRAS, previamente construído e validado quanto ao seu conteúdo, conceitos teóricos, função discriminante e confiabilidade ${ }^{(11)}$ sendo composto por quatro indicadores de processo e estrutura, a saber: 1. Estrutura do PPCIRAS (PCET): avalia sua estrutura, incluindo formação e suporte técnico-operacional, tais como recursos humanos, infraestrutura física e instrumentos técnicos e administrativos, com 10 componentes de avaliação; 2. Diretrizes operacionais de IRAS (PCDO): avalia a existência de diretrizes operacionais de áreas ou serviços hospitalares, nas formas de manuais, normas e procedimentos operacionais elaboradas ou incorporadas ao PPCIRAS, com 15 componentes de avaliação; 3. Vigilância epidemiológica de IRAS (PCVE): avalia se os PPCIRAS executam um sistema de vigilância epidemiológica, por meio de atividades que incluem busca ativa, perfil de indicadores epidemiológicos, identificação e notificação de casos de IRAS, com 10 componentes de avaliação; e 4. Atividades de prevenção e controle de IRAS (PCCP): avalia as atividades de prevenção e controle de IRAS nos vários serviços ou setores hospitalares, incluindo inspeção, orientações e avaliações de diretrizes introduzidas, participação em reuniões dos setores, realização de consultas e esclarecimentos cotidianos por ocasião de demandas espontâneas, entre outras, com 14 componentes de avaliação. Cada indicador descreve sua fundamentação teórica de evidência e orienta o processo de avaliação, bem como a fórmula para estabelecer o índice de conformidade ${ }^{(11)}$.

\section{ANÁlISE E TRATAMENTO DOS DADOS}

O desfecho avaliado foi o Índice de Conformidade (IC), composto pela proporção de itens em conformidade em relação ao número de itens avaliados. O IC $\geq 70 \%$ foi definido como bom nível de qualidade para estrutura e processos dos PPCIRAS. A codificação e 
a entrada de dados foram realizadas com auxílio do software Microsoft Excel (2015) e do programa Statistical Package for Social Sciences (SPSS 22). Foram calculados os percentis da distribuição das pontuações atingidas nos ICs. Associações entre variáveis selecionadas e o IC foram analisadas com teste exato de Fisher a um nível de significância de 5\%.

\section{Aspectos éticos}

A pesquisa respeitou os princípios éticos e legais contidos na Resolução no 466/2012, do Conselho Nacional de Saúde e foi previamente aprovada pelo Comitê de Ética em Pesquisa da Escola de Enfermagem da Universidade de São Paulo em 02/07/2015, sob o parecer no 1.212.774. O Termo de Consentimento Livre e Esclarecido (TCLE) foi previamente assinado pelos participantes envolvidos.

\section{RESULTADOS}

Dentre os 27 hospitais recrutados, 14 consentiram em participar, correspondendo a $52 \%$ da amostra total de acesso. As razões para a perda da amostra foram: ausência de resposta do hospital para participação após cinco tentativas $(n=5)$, recusa em participar $(n=7)$ e inexistência de PPCIRAS atuante $(n=1)$.

Os hospitais apresentavam em média 30,7 leitos (DP 14,1; mediana 30,0 leitos). A amostra foi composta por 11 hospitais privados $(78,6 \%)$ e 3 públicos $(21,4 \%)$. A maioria desses hospitais possuía assistência geral $(71,4 \%)$ e aqueles especializados $(28,6 \%)$ atendiam geriatria, pediatria e cirurgias reparadoras/plásticas.

Somente 42,9\% possuíam laboratório de análises próprio. Esse serviço era terceirizado na maioria dos hospitais; contudo, as três instituições públicas participantes possuíam laboratório próprio. $\mathrm{O}$ programa para cirurgia segura, em consonância com diretrizes nacionais ${ }^{(12)}$, estava implantado em sete hospitais $(50,0 \%)$.

Indicadores epidemiológicos eram utilizados por nove $(64,3 \%)$ dos hospitais estudados. Mais frequentemente, esses indicadores eram as taxas de infecções em cirurgias limpas $(n=9)$ e partos cesáreos $(n=8)$. Pouco mais da metade $(n=9)$ utilizava o método ativo para a busca de infecções. A maioria dos resultados da vigilância resultavam em relatórios apenas com informações estatísticas descritivas (71,4\%), sem análise e interpretação (64,3\%) ou recomendações $(57,1 \%)$. A disseminação das informações epidemiológicas para a comunidade hospitalar ou comunicação para autoridades sanitárias não era realizada em 7 (50,0\%) hospitais.

Os hospitais que possuíam centro cirúrgico apresentavam em média duas salas cirúrgicas (DP 1,0). A média mensal de cirurgias realizadas nas instituições foi 115. As Comissões de Controle de Infecção Hospitalar (CCIH) foram implementadas nos hospitais desse estudo a partir de 2007.

Evidenciou-se grande variação nos IC dos PPCIRAS (Figura 1). Os menores IC foram observados nos indicadores de estrutura do PPCIRAS e de vigilância epidemiológica. Houve maior dispersão dos dados observados no que se referiu ao indicador de vigilância epidemiológica das IRAS. Quanto ao IC de estrutura dos PPCIRAS, verificou-se que a mediana dos hospitais não atingiu o IC de 70\%

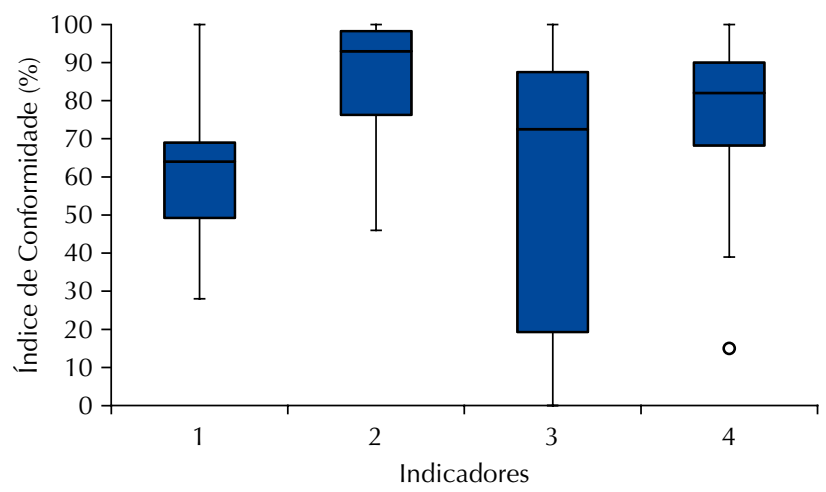

1 = Estrutura do PCIRAS; 2 = Diretrizes Operacionais do PCIRAS 3 = Vigilância Epidemiológica de IRAS; 4 = Atividades de Prevenção e Controle de IRAS

Figura 1 - Índice de Conformidade dos hospitais segundo os indicadores de avaliação dos Programas de Prevenção e Controle de Infecções Relacionadas a Assistência à Saúde (PPCIRAS) - Região de Taubaté, SP, Brasil, 2015-2016.

As instituições privadas apresentaram maiores IC em relação às públicas (Tabela 1), porém esta diferença não foi significativa $(\mathrm{p}>0.05)$. As instituições que possuem Unidade de Terapia Intensiva (UTI) apresentaram melhores IC quando comparadas aquelas sem UTI (Tabela 2).

Tabela 1 - Distribuição do índice de conformidade referente aos indicadores dos PPCIRAS em hospitais públicos e privados - Região de Taubaté, SP, Brasil, 2015-2016.

\begin{tabular}{|c|c|c|c|c|c|c|c|c|}
\hline \multirow{2}{*}{ Indicadores } & \multicolumn{4}{|c|}{$\begin{array}{l}\text { Hospitais públicos } \\
(\mathrm{N}=3)\end{array}$} & \multicolumn{4}{|c|}{$\begin{array}{l}\text { Hospitais privados } \\
(\mathrm{N}=11)\end{array}$} \\
\hline & Média (DP) & Mín. & Med. & Máx. & Média (DP) & Mín. & Med. & Máx. \\
\hline Estrutura do PPCIRAS & $48,7(17,8)$ & 28,2 & 59,0 & 59,0 & $64,3(20,4)$ & 28,2 & 69,2 & 100,0 \\
\hline Diretrizes operacionais do PPCIRAS & $81,3(19,9)$ & 58,3 & 92,3 & 93,3 & $85,4(19,3)$ & 46,2 & 93,3 & 100,0 \\
\hline Vigilância epidemiológica de IRAS & $26,7(46,2)$ & 0,0 & 0,0 & 80,0 & $66,4(35,0)$ & 0,0 & 77,8 & 100,0 \\
\hline Atividades de prevenção e controle de IRAS & $56,6(36,9)$ & 15,4 & 67,7 & 86,7 & $79,4(19,0)$ & 38,9 & 85,2 & 100,0 \\
\hline
\end{tabular}

PPCIRAS: Programas de Prevenção e Controle de Infecções Relacionadas à Assistência à Saúde; DP: Desvio Padrão; Mín: Mínimo; Med: Mediana; Máx: Máximo. IRAS - Infecções Relacionadas à Assistência à Saúde. 
Tabela 2 - Estatísticas descritivas do índice de conformidade dos indicadores dos PPCIRAS segundo presença de UTI - Região de Taubaté, SP, Brasil, 2015-2016.

\begin{tabular}{|c|c|c|c|c|c|c|c|c|c|c|}
\hline \multirow{2}{*}{ Indicadores } & \multicolumn{5}{|c|}{ Hospitais com UTI (N=3) } & \multicolumn{5}{|c|}{ Hospitais sem UTI $(\mathrm{N}=11)$} \\
\hline & M & Mín. & Med & Máx. & IC $\$ 95 \%$ & M & Mín. & Med & Máx. & IC $\$ 95 \%$ \\
\hline Estrutura do PPCIRAS* & 83,0 & 69,2 & 79,5 & 100,0 & $81,8-84,0$ & 55,0 & 28,2 & 59,0 & 79,5 & $54,6-55,4$ \\
\hline Diretrizes operacionais do PPCIRAS & 95,6 & 93,3 & 93,3 & 100,0 & $94,5-96,6$ & 81,5 & 46,2 & 92,3 & 100,0 & $81,1-81,9$ \\
\hline Vigilância epidemiológica de IRAS** & 93,3 & 80,0 & 100,0 & 100,0 & $91,1-95,5$ & 48,2 & 0,0 & 60,0 & 100,0 & $47,4-49,0$ \\
\hline Atividades de Prevenção e controle IRAS & 89,1 & 76,0 & 91,3 & 100,0 & $87,8-90,4$ & 70,5 & 15,4 & 79,0 & 100,0 & $70,0-71,0$ \\
\hline
\end{tabular}

PPCIRAS - Programas de Prevenção e Controle de Infecções Relacionadas à Assistência à Saúde; M: Média; Mín: Mínimo; Med: Mediana; Máx: Máximo. IRAS - Infecções Relacionadas à Assistência à Saúde. IC $\$ 95 \%$ Índice de Confiança 95\%. ${ }^{*} p=0,03$. ${ }^{* *} p=0,04$.

Apesar dos hospitais possuírem enfermeiros designados para PPCIRAS ( $n=13)$, somente em três das instituições estes atuavam com dedicação exclusiva de seis horas diárias no mínimo. O tempo de dedicação diária exclusiva ao Serviço de Controle de Infecção Hospitalar (SCIH) foi em média 3,3 horas. Em sua maioria $(n=9)$, os enfermeiros possuíam experiência em PPCIRAS inferior a 2 anos; contudo os médicos eram em geral mais experientes, com dois anos ou mais $(n=6)$. Somente 2 dos enfermeiros do SCIH possuíam especialização em prevenção de IRAS e 3 haviam realizado cursos de curta duração (ex.: 30 horas).

As diretrizes operacionais contendo recomendações para prevenção e controle de IRAS estavam presentes em todos os hospitais ( $n=14)$, com exceção para as recomendações de lavagem de roupas, as quais estavam presentes em somente 8 hospitais. Contudo, observou-se que a maioria das instituições $(n=7)$ utilizava serviço terceirizado de lavanderia.

Em relação às atividades desenvolvidas para a prevenção e controle de IRAS, foi observado que se direcionavam às unidades de maior risco, tais como a UTI, a central de esterilização e o centro cirúrgico. Poucos PPCIRAS demonstraram ter alguma atuação documentada em relação aos serviços terceirizados, como laboratórios $(\mathrm{n}=7)$ e unidades de diálise $(\mathrm{n}=0)$.

\section{DISCUSSÃO}

PPCIRAS bem estruturados podem reduzir taxas infecciosas e, por conseguinte, a carga econômica e social gerada por esses eventos ${ }^{(13)}$. Apesar de obrigatório no Brasil desde $1997^{(14)}$, o PPCIRAS ainda está longe do desejável, principalmente para os pequenos hospitais ${ }^{(15)}$ que lidam com a falta de recursos humanos, financeiros e estruturais para estabelecerem a prevenção de IRAS de maneira sistematizada. Sendo assim, este estudo vem a contribuir para o reconhecimento do cenário dos PPCIRAS de pequenos hospitais no país. Na região estudada, esses PPCIRAS se mostraram ainda incipientes.

No Brasil, os pequenos hospitais se caracterizam pelo predomínio de atividade cirúrgica e obstétrica. Esse fato aponta para uma provável alta incidência de infecção de sítio cirúrgico, considerando os dados disponíveis para este grupo de hospitais até o presente momento ${ }^{(8)}$. Este fato também foi destacado por um estudo realizado no interior paulista que identificou uma média mensal de 5 dessas infecções para cada hospital de pequeno porte ${ }^{(16)}$. Considerando a proporção de hospitais de pequeno porte no país e, extrapolando estas estimativas, pode-se assumir que um grande número de pessoas no Brasil é afetado, o que justifica a necessidade de estudos com foco neste grupo de instituições.

A terceirização de serviços de microbiologia em muitas instituições foi também observada em outros estudos nacionais ${ }^{(15,17)}$. Este fato alerta para a possibilidade de empecilhos para as ações da CCIH, que dependem de uma ação qualificada e oportuna, no que tange aos resultados de exames microbiológicos. Estudos realizados em outras localidades do país ${ }^{(2,18)}$ também apontaram deficiências nas práticas de prevenção e controle de IRAS em serviços terceirizados, como de lavanderia e laboratório.

No presente estudo, observou-se uma atuação em atividades relacionadas à segurança do paciente em uma parcela das instituições, principalmente no que tange a cirurgia segura. No entanto, de modo geral, evidenciou-se certa fragilidade no alcance das ações dos PPCIRAS em diversos aspectos. Notadamente, seria esperado que a busca ativa de casos de IRAS estivesse implementada em todas as instituições, como recomendado pela Organização Mundial de Saúde (OMS) ${ }^{(19)}$, o que não foi observado.

A efetiva retroalimentação aos profissionais quanto ao dados epidemiológicos é um dos componentes considerados como essenciais pela $\mathrm{OMS}^{(19)}$. Contudo, nestes hospitais verificou-se a ausência de adequada análise e a disseminação limitada das informações epidemiológicas como mecanismos de melhoria nos processos de prevenção. Esse fato pode estar relacionado à pequena carga horária destinada ao PPCIRAS ou a falta de especialização dos profissionais para atuar nesta área. Como já observado por outros autores, muitos profissionais atuam como meros coletores de dados $\mathrm{e}$ não possuem proatividade e liderança necessárias para intervenções de melhoria ${ }^{(20)}$.

Por outro lado, em instituições de pequeno porte, os enfermeiros na maioria das vezes necessitam dividir sua carga horaria com outras atividades, incluindo a assistência direta ou funções administrativas. Outros estudos também evidenciaram essa problemática: um estudo canadense constatou déficit de carga horária em 77,4\% das atividades dedicadas ao PPCIRAS ${ }^{(21)}$; assim como, um levantamento realizado em nosso país evidenciou recursos humanos inadequados em $83 \%$ do PPCIRAS ${ }^{(22)}$. Nos pequenos hospitais rurais, os profissionais que atuam com prevenção e controle de IRAS são mais propensos a ter vários empregos, responsabilidades e dispender tempo diário menor nesse trabalho ${ }^{(9)}$, assim como, pode ocorrer também em hospitais 
urbanos que apresentam inadequado dimensionamento de recursos humanos para PPCIRAS em relação à carga horária mínima recomendada ${ }^{(23)}$. A relação entre capacidade de leitos e tempo de trabalho exclusivo nos PPCIRAS ainda é um tema controverso em diversos países, principalmente para os pequenos hospitais ${ }^{(7,9,23-25)}$.

A rotatividade de recursos humanos, o tempo empregado no treinamento de novos funcionários, as limitações na competência de linguagem e a pequena carga horária de trabalho permeiam as barreiras existentes para a implementação de um PPCIRAS eficaz ${ }^{(26)}$. No entanto, equipes bem estruturadas, direcionadas e com um clima institucional que prioriza a prevenção são facilitadores para o mapeamento de tarefas e alocação de recursos humanos na instituição ${ }^{(26)}$. Isto é particularmente importante em instituições com recursos limitados para implementação de intervenções eficientes para a prevenção de IRAS.

O mesmo instrumento de avaliação utilizado em nosso estudo foi também aplicado em diversas regiões do Brasil, apresentando grandes variações nos resultados ${ }^{(2,11,15,18,27)}$. Estudo realizado na cidade de São Paulo, verificou melhores resultados, com IC superiores a $80 \%$, com destaque para os indicadores de estrutura do PPCIRAS e o de vigilância epidemiológica, os quais alcançaram IC muito próximos a $99 \%{ }^{(11)}$. Outro estudo semelhante, na cidade de Ribeirão Preto, evidenciou IC para os indicadores de estrutura do PPCIRAS $(75,4 \%)$, diretrizes operacionais $(59,4 \%)$, vigilância epidemiológica $(83,7 \%)$ e atividades de prevenção e controle de IRAS $(56,8 \%)^{(18)}$. Destacando que o desempenho dos PPCIRAS de Ribeirão Preto, em sua maioria constituído por pequenos hospitais, divergiu do presente estudo, uma vez que os maiores IC foram em estrutura e vigilância epidemiológica dos PPCIRAS. Já no estado do Paraná, os IC observados foram inferiores: o indicador de estrutura do PPCIRAS apresentou IC igual a 79,4\%, seguido do indicador de atividades de vigilância epidemiológica, $76,0 \%{ }^{(2)}$. Os demais indicadores obtiveram IC abaixo de $70 \%$, sendo $65,5 \%$ para o indicador de diretrizes operacionais e $63,2 \%$ para o de atividades de prevenção e controle de $\operatorname{IRAS}^{(2)}$. Um estudo realizado em Campo Grande evidenciou IC superiores a 80,0\% para os indicadores de estrutura do PPCIRAS e vigilância epidemiológica, porém IC próximos a $60,0 \%$ em diretrizes operacionais e atividades de prevenção e controle de $\operatorname{IRAS}^{(27)}$.

Estudo brasileiro randomizado de abrangência nacional que utilizou o mesmo instrumento de avaliação, apontou que hospitais com menos de 50 leitos apresentaram valores de IC bem inferiores quando comparados aos hospitais de maior porte, como os maiores de 200 leitos $^{(15)}$. O mesmo estudo também evidenciou que muitos PPCIRAS não baseiam seus esforços preventivos em dados de vigilância e priorizam as das atividades preventivas para as unidades de cuidados intensivos ${ }^{(15)}$. Isto corrobora os achados do presente estudo que apontam para o fato de que a existência de UTIs parece favorecer melhores IC. Contudo, este dado deve ser interpretado com cautela devido ao tamanho da amostra de serviços de UTI em nossa casuística.
A variabilidade observada nos diferentes estudos citados é de certa forma esperada, uma vez que o país é bastante heterogêneo em relação a características sociais, econômicas e culturais. É importante ressaltar que dentre os estudos identificados apenas $\mathrm{um}^{(15)}$ apresentou resultados individualizados de acordo com o porte hospitalar. Este aspecto torna-se importante, pois resultados de avaliação de conformidade dos PPCIRAS obtidos com hospitais de grande porte podem não refletir a realidade dos pequenos hospitais. Diversas são as razões que impactam de maneira positiva ou negativa nestas diferenças, entre elas podemos citar as características: a capacidade limitada de recursos humanos e materiais, atenção predominantemente cirúrgica e com menor número de casos de terapia intensiva e a menor frequência de uso de dispositivos e procedimentos invasivos ${ }^{(8)}$.

Além disso, no estudo realizado em Campo Grande foram destacados IC discrepantes entre as instituições da mesma região estudada, o que reforça a existência de grande heterogeneidade também entre as realidades de prevenção e controle de IRAS nos hospitais de uma mesma região no país ${ }^{(27)}$, corroborando com os achados da presente pesquisa.

No presente estudo não foi possível identificar diferenças significantes entre o financiamento privado e público das instituições avaliadas, no que tange ao IC dos PPCIRAS. Um estudo brasileiro realizado no Rio Grande do Norte, buscou avaliar e comparar a aderência às práticas de prevenção de IRAS de acordo com o tipo de administração hospitalar (privada, pública com administração direta, pública com administração indireta), porém sem resultados conclusivos quanto às diferenças ${ }^{(28)}$. Este é um aspecto importante, que ainda tem sido pouco estudado e que deve merecer atenção dos pesquisadores no futuro.

A falta de cumprimento das ações preventivas aponta para a necessidade de medidas de educação e auditoria dos processos, que por sua vez, requerem uma estrutura mínima. É compreensível que os hospitais de grande porte e de ensino liderem a pesquisa para a prevenção e controle de IRAS no mundo em desenvolvimento. Entretanto, para melhorarmos a qualidade e a segurança da assistência prestada, necessitamos que a política de saúde seja reformulada, considerando os recursos disponíveis e as prioridades de ações para os pequenos hospitais, sejam eles, rurais ou urbanos.

A preocupação com a temática de prevenção de IRAS em pequenos hospitais, contudo, não aflige apenas países em desenvolvimento. $\mathrm{Na}$ Austrália, por exemplo, verificou-se que há grande número de pequenos hospitais no estado de Victoria. São 90 pequenos hospitais públicos de cuidados agudos em comparação a apenas 28 hospitais de maior porte. Em virtude dessa realidade, foi desenvolvido o Victorian Hospital-Acquired Infection Surveillance System (VICNISS), que tem como objetivo desenvolver, implementar, apoiar e avaliar programas de vigilância em todo o estado, a fim de otimizar o desempenho na redução das $\operatorname{IRAS}^{(29)}$. Este programa utiliza indicadores de processo e de resultados e possui estudos com foco em pequenos hospitais ${ }^{(10,29)}$.

O nível de esforço necessário para manter um programa eficaz e eficiente em termos de custo para os pequenos hospitais é matéria de discussão. A ausência de índices de IRAS 
comparáveis entre os pequenos hospitais é um grande dificultador para análises da qualidade da assistência. Em decorrência, há falsas premissas de que seus índices são baixos quando comparados aos dados de referência de hospitais de maior porte. Para contornar está dificuldade, alguns autores sugerem aumentar o período de coleta de dados para análise ou reportar apenas eventos sentinelas ${ }^{(7,29)}$. Entretanto, não há consenso quanto ao melhor sistema de vigilância a ser adotado para este tipo de hospitais.

Muitos dos pequenos hospitais despendem recursos razoáveis para desenvolver seus PPCIRAS, na maioria das vezes baseados em modelos de hospitais maiores e seguindo recomendações e consenso de especialistas. Mesmo as recomendações atuais da OMS, são baseadas em evidências em sua maioria produzidas em países desenvolvidos e em hospitais de maior porte ${ }^{(19)}$. Contudo, há uma ampla diversidade entre a realidade dos pequenos hospitais em todo o mundo, de modo que se torna difícil definir quais sejam as recomendações custo-efetivas para este grupo de instituições.

Os achados do presente estudo reforçam que, ainda no Brasil, a prevenção e controle de IRAS se encontra muito aquém para estas instituições. Tais resultados trazem contribuição para o reconhecimento do cenário atual, no qual atuam enfermeiros engajados na prevenção e controle de IRAS em pequenos hospitais, e os desafios a serem superados por eles na liderança de suas equipes. Um maior conhecimento do contexto pode abrir caminhos para o avanço da enfermagem para melhorias na qualidade da assistência e segurança do paciente, atuando como importante protagonista no processo político ${ }^{(30)}$.

Um ponto forte do presente estudo foi a coleta de dados realizada in loco por profissional com experiência em controle de infecção, minimizando o viés de coleta de dados. Entre as suas limitações, houve recusa de metade das instituições elegíveis em participar da avaliação. Contudo, como desconhecemos as razões para a recusa, não é possível estimar com maior precisão se estas razões podem ter produzido um viés favorável ou desfavorável em relação aos IC observados. Outra limitação potencial seria a exclusão inadvertida de hospitais não cadastrados pelo CNES em virtude de atualizações periódicas. A seleção da região foi uma decisão pragmática relevante levando em consideração as dificuldades operacionais para a realização desse estudo, tendo em vista que o Brasil possui grandes dimensões geográficas e recursos limitados.

Os resultados obtidos no presente estudo sugerem que ainda existe uma insuficiente atenção das autoridades e gestores na saúde para assegurar a homogeneidade dos PPCIRAS nos hospitais e minimizar as desigualdades na qualidade da atenção em saúde na região.

\section{CONCLUSÃO}

O presente estudo demonstrou importante variação dos IC de PPCIRAS em uma amostra de hospitais de uma região do estado de São Paulo. Dentre os quatro indicadores verificados no presente estudo, apenas o indicador referente às diretrizes operacionais do PPCIRAS esteve acima de 90\% na mediana dos hospitais avaliados. $\mathrm{O}$ indicador que apontou maior dispersão dos resultados de IC entre os hospitais estudados foi referente a vigilância epidemiológica das IRAS.

Estes dados apontam para a necessidade de outros estudos que avaliem a efetividade de programas a fim de identificar os melhores modelos para a prevenção e controle de IRAS compatíveis com as características de pequenos hospitais.

\section{RESUMO}

Objetivo: Avaliar a conformidade de estrutura e processo dos programas de prevenção e controle de infecções relacionadas à assistência à saúde. Método: Estudo prospectivo, transversal, realizado de 2015 a 2016, em pequenos hospitais de até 70 leitos de uma região do estado de São Paulo. Foram avaliados 4 indicadores previamente validados, expressos em índice de conformidade (porcentagem em relação aos itens avaliados). Resultados: Dentre os 27 hospitais recrutados, 14 consentiram em participar. Os valores médios de conformidade para cada indicador foram: Estrutura dos programas 61,0\%; Diretrizes operacionais 84,5\%; Vigilância epidemiológica 57,9\%; Atividades de prevenção 74,5\%. Maior conformidade foi observada em hospitais privados (73,9\%) e com presença de unidade de terapia intensiva (90,3\%). Os hospitais possuíam enfermeiros designados para o programa (92,9\%), mas somente $23,1 \%$ das instituições privadas atuavam com dedicação exclusiva de seis horas. Conclusão: Apenas o indicador referente às Diretrizes Operacionais dos programas avaliados esteve acima de $90 \%$ de conformidade na mediana dos hospitais. A maior dispersão dos resultados de conformidade entre os hospitais estudados foi referente ao indicador de Vigilância Epidemiológica.

\section{DESCRITORES}

Infecção Hospitalar; Controle de Infecções; Indicadores Básicos de Saúde; Avaliação em Saúde.

\section{RESUMEN}

Objetivo: Evaluar la conformidad de estructura y proceso de los programas de prevención y control de infecciones relacionados a la asistencia a la salud. Método: Estudio prospectivo, transversal, realizado de 2015 a 2016, en pequeños hospitales de hasta 70 camas de una región del estado de São Paulo. Fueran evaluados 4 indicadores previamente validados, expresos en índice de conformidad (porcentaje en relación a los ítems evaluados). Resultados: Dentro los 27 hospitales reclutados, 14 consentirán en participar. Los valores medios de conformidad para cada indicador fueran: Estructura de los programas 61,0\%; Directrices operacionales 84,5\%; Vigilancia epidemiológica 57,9\%; Actividades de prevención 74,5\%. Mayor conformidad fue observada en hospitales privados $(73,9 \%)$ y con presencia de la unidad de terapia intensiva (90,3\%). Los hospitales poseían enfermeros designados para o programa (92,9\%), mas solamente $23,1 \%$ de las instituciones privadas actuaban con dedicación exclusiva de seis horas. Conclusión: Apenas el indicador referente a las Directrices Operacionales de los programas evaluados estuvo arriba de $90 \%$ de conformidad en la media de los hospitales. La mayoría de la dispersión de los resultados de conformidad entre los hospitales estudiados fue referente al indicador de Vigilancia Epidemiológica.

\section{DESCRIPTORES}

Infección Hospitalaria; Control de Infecciones; Indicadores de Salud; Evalución en Salud. 


\section{REFERÊNCIAS}

1. Lamarsalle L, Hunt B, Schauf M, Szwarcensztein K, Valentine WJ. Evaluating the clinical and economic burden of healthcare-associated infections during hospitalization for surgery in France. Epidemiol Infect. 2013;141(12):2473-82. DOI: 10.1017/S0950268813000253

2. Alves DCl, Lacerda RA. Evaluation of Programs of Infection Control related to healthcare assistance in hospitals. Rev Esc Enferm USP. 2015;49(n.spe):65-73. DOI: http://dx.doi.org/10.1590/S0080-623420150000700010

3. Price L, MacDonald J, Melone L, Howe T, Flowers P, Currie K, et al. Effectiveness of national and sub-national infection prevention and control interventions in high-income and upper-middle income countries: outcomes of a systematic review. Lancet Infect Dis. 2018;18(5):e159-71. DOI: 10.1016/S1473-3099(17)30479-6

4. Uga MAD, López EM. Os hospitais de pequeno porte e sua inserção no SUS. Ciênc Saúde Coletiva. 2007;12(4):915-28. http://dx.doi.org/10.1590/S1413-81232007000400013

5. Scherbaum M, Kösters K, Mürbeth RE, Ngoa UA, Kremsner PG, Lell B, et al. Incidence, pathogens and resistance patterns of nosocomial infections at a rural hospital in Gabon. BMC Infect Dis. 2014;14:124. DOI: 10.1186/1471-2334-14-124

6. Kuntz JL, Cavanaugh JE, Becker LK, Ward MA, Appelgate DM, Herwaldt LA, et al. Clostridium difficile - associated disease in patients in a small rural hospital. Infect Control Hosp Epidemiol. 2007;28(11):1236-9. DOI: 10.1086/521662

7. Stevenson KB, Murphy CL, Samore MH, Hannah EL, Moore JW, Barbera J, et al. Assessing the status of infection control programs in small rural hospitals in the western United States. Am J Infect Control. 2004;32(5):255-61. DOl: 10.1016/j.ajic.2003.10.016

8. Fortaleza CMCB, Padoveze MC, Kiffer CRV, Barth AL, Carneiro ICDRS, Giamberardino HIG, et al. Multi-state survey of healthcare-associated infections in acute care hospitals in Brazil. J Hosp Infect. 2017;96(2):139-44. DOI: 10.1016/j.jhin.2017.03.024

9. Reese SM, Gilmartin H, Rich KL, Price CS. Infection prevention needs assessment in Colorado hospitals: rural and urban settings. Am J Infect Control. 2014;42(6):597-601. DOI: 10.1016/J.AJIC.2014.03.004

10. Bennett NJ, Bull AL, Dunt DR, Gurrin LC, Richards MJ, Russo PL, et al. A profile of smaller hospitals: planning for a novel, statewide surveillance program, Victoria, Australia. Am J Infect Control. 2006;34(4):170-5.

DOI: 10.1016/j.ajic.2005.05.011

11. Silva CPR, Lacerda RA. Validation of a proposal for evaluating hospital infection control programs. Rev Saúde Pública. 2011;45(1):121-8. DOI: http://dx.doi.org/10.1590/S0034-89102010005000052

12. Brasil. Ministério da Saúde. Portaria n. 529, de 01 de abril de 2013. Institui o Programa Nacional de Segurança do Paciente (PNSP) [Internet]. Brasília; 2013 [citado 2019 jan. 20]. Disponível em: http://www.saude.mt.gov.br/upload/controle-infeccoes/pasta2/portariamsgm-n-529-de-01-04-2013.pdf

13. Raschka S, Dempster L, Bryce E. Health economic evaluation of an infection prevention and control program: are quality and patient safety programs worth the investment? Am J Infect Control. 2013;41(9):773-7. DOI: 10.1016/j.ajic.2012.10.026

14. Brasil. Lei n. 9.431, de 06 de janeiro de 1997. Dispõe sobre a obrigatoriedade de manutenção de programas de controle de infecção hospitalar pelos hospitais do país. Brasília [Internet]; 1997 [citado 2019 jan. 20]. Disponível em: http://www.planalto.gov.br/ccivil_03/leis/l9431.htm

15. Padoveze MC, Fortaleza CMCB, Kiffer C, Barth AL, Carneiro ICR, Giamberardino HIG, et al. Structure for prevention of health care-associated infections in Brazilian hospitals: A countrywide study. Am J Infect Control. 2016;44(1):74-9. DOI: 10.1016/j.ajic.2015.08.004

16. Armede VCB, Abraão LM, Fortaleza CMCB. Surgical site infections in very small hospitals in inner Brazil: unveiling a relevant issue for developing countries. Am J Infect Control. 2017;45(8):935-6. DOI: 10.1016/j.ajic.2017.04.289

17. Costa LB, Cardoso MRA, Ferreira CG, Levy CE, Borba HM, Sallas J, et al. National prevalence survey in Brazil to evaluate the quality of microbiology laboratories: the importance of defining priorities to allocate limited resources. Rev Panam Salud Publica. 2013;33(1):73-8. DOI: 10.1590/s1020-49892013000100010

18. Meneguetti MG, Canini SRMS, Belissímo-Rodrigues F, Laus AM. Avaliação dos programas de controle de infecção hospitalar em serviços de saúde. Rev Latino Am Enfermagem. 2013;23(1):98-105. DOI: http://dx.doi.org/10.1590/0104-1169.0113.2530

19. Storr J, Twyman A, Zingg W, Damani N, Kilpatrick C, Reilly J, et al. Core components for effective infection prevention and control programmes: new WHO evidence-based recommendations. Antimicrob Resist Infect Control. 2017;6:6. DOI: 10.1186/s13756-016-0149-9

20. Murphy D, Carrico R, Warye K. Building the infection prevention system of tomorrow: proceedings of the 2007 APIC Futures Summit. Am J Infect Control. 2008;36(4):232-40. DOI: 10.1016/J.AJIC.2007.11.001

21. Zoutman DE, Ford BD. A comparison of infection control program resources, activities, and antibiotic resistant organism rates in Canadian acute care hospitals in 1999 and 2005: pre- and post-severe acute respiratory syndrome. Am J Infect. Control 2008;36(10):711-7. DOI: 10.1016/J.AJIC.2008.02.008

22. Brasil. Ministério da Saúde; Agência Nacional de Vigilância Sanitária. Programa Nacional de Prevenção e Controle de infecções Relacionadas à Assistência à Saúde (2013-2015)]. Brasília; 2013.

23. Daltoé T, Breier A, Santos HB, Wagner MB, Kuchenbecker RS. Serviços de controle de infecção hospitalar: características, dimensionamento e atividades realizadas. Rev Soc Bras Clin Med [Internet]. 2014 [citado 2019 jan. 20];12(1):35-45. Disponível em: http://files.bvs.br/upload/S/1679-1010/2014/v12n1/a4041.pdf

24. Stone PW, Dick A, Pogorzelska MPH, Horan TC, Furuya EY, Larson E. Staffing and structure of infection prevention and control programs. Am J Infect Control. 2009;37(5):351-7. DOI: 10.1016/j.ajic.2008.11.001

25. Van den Broek PJ, Kluytmans JA, Ummels LC, Voss A, Vandenbroucke-Grauls CM. How many infection control staff do we need in hospitals? J Hosp Infect 2007;65(2):108-11. DOI: 10.1016/j.jhin.2006.10.003

26. Barker AK, Brown K, Siraj D, Ahsan M, Sengupta S, Safdar N. Barriers and facilitators to infection control at a hospital in northern India: a qualitative study. Antimicrob Resist Infect Control. 2017;6:35. DOI: 10.1186/s13756-017-0189-9

27. Giroti ALB, Ferreira AM, Rigotti MA, Sousa AFL, Frota OP, Andrade D. Hospital infection control programs: assessment of process and structure indicators. Rev Esc Enferm USP. 2018;52:e03364. DOI: http://dx.doi.org/10.1590/s1980-220x2017039903364 
28. Gama ZAS, Hernández PJS, Freitas MR, Padoveze MC, Saraiva COPO, Paulino LG, et al. Good infections prevention practices in three Brazilian hospitals: implications for patients safety policies. J Infect Public Health. 2019;12(5):619-24. DOI: 10.1016/j.jiph.2019.02.016

29. Bennett NJ, Bull AL, Dunt DR, Spelman DW, Russo PL, Richards MJ. Implementation of a pilot surveillance program for smaller acute care hospitals. Am J Infect Control. 2007;35(3):196-9. DOI: 10.1016/j.ajic.2006.04.209

30. Lamblet LCR, Padoveze MC. Comissões de controle de infecção hospitalar: perspectiva de ações do Conselho Regional de Enfermagem. Cad Ibero Am Dir Sanit. 2018;7(1):29-42. DOI: http;//dx.doi.org/10.17566/ciads.v7i1.426 